\title{
openheart Gated metabolic myocardial imaging, a surrogate for dual perfusion- metabolism imaging by positron emission tomography
}

\author{
Abdallah AIMohammad, ${ }^{1}$ Murdoch Y Norton, ${ }^{2}$ Andrew E Welch, ${ }^{2}$ Peter F Sharp, ${ }^{2}$ \\ Stephen Walton ${ }^{3}$
}

To cite: AlMohammad A, Norton MY, Welch AE, et al. Gated metabolic myocardial imaging, a surrogate for dual perfusion-metabolism imaging by positron emission tomography. Open Heart 2017;4:e000581. doi:10.1136/ openhrt-2016-000581

Received 2 December 2016 Revised 17 April 2017 Accepted 13 June 2017

\section{CrossMark}

${ }^{1}$ South Yorkshire Cardiothoracic Centre, Chesterman Wing, Northern General Hospital, Sheffield, UK

${ }^{2}$ The Department of Medical Physics and Bio-medical Engineering, Aberdeen University, Aberdeen, UK ${ }^{3}$ The Department of Cardiology, Aberdeen Royal Infirmary, Grampian University Hospitals NHS Trust, Aberdeen, UK

Correspondence to Dr Abdallah AlMohammad, South Yorkshire Cardiothoracic Centre, Chesterman Wing, Northern General Hospital, Herries Road, Sheffield S5 7AU, UK;; a.al.mohammad.87@ googlemail.com

\section{ABSTRACT}

Objective Perfusion-metabolism mismatch pattern on positron emission tomography (PET) predicts hibernating myocardium. We assess the ECG-gated metabolic PET as a surrogate for the perfusion-metabolism mismatch pattern on PET imaging.

Methods ${ }^{13} \mathrm{~N}$-Ammonia $\left(\mathrm{NH}_{3}\right)$ and ${ }^{18} \mathrm{~F}$-fluorodeoxyglucose (FDG) are respectively perfusion and metabolism PET tracers. We used ECG gating to acquire FDG-PET to collect wall thickening (mechanical) data. These allow detection of metabolic activity in regions with reduced contraction (metabolism-mechanical mismatch pattern). We had two data sets on each patient: perfusion-metabolism and metabolism-mechanical data sets. We tested the hypothesis that metabolism-mechanical pattern on PET could predict perfusion-metabolism mismatch pattern. Results We studied 55 patients (48 males), mean age 62 years. All were in sinus rhythm, and had impaired left ventricular contraction. Perfusion-metabolism mismatch pattern was found in 26 patients. Metabolism-mechanical mismatch pattern was found in 25 patients. The results were concordant in 52 patients (95\%). As a surrogate for perfusion-metabolism mismatch pattern, demonstration of metabolism-mechanical mismatch pattern is highly sensitive (92\%) and specific (97\%). In this cohort, the positive and negative predictive accuracy of the new method are $96 \%$ and $93 \%$, respectively.

Conclusion Metabolism-mechanical mismatch pattern could predict perfusion-metabolism mismatch pattern in patients with myocardial viability criteria on PET. Prospective validation against the gold standard of improved myocardial contraction after revascularisation is needed.

\section{INTRODUCTION}

It is important to predict the presence of viable and potentially hibernating myocardium preoperatively. This stems from increased mortality rates of the patients with heart failure, ${ }^{1}$ the relatively high prevalence of viability in those with severe left ventricular impairment $^{2}$ and their improved prognosis following revascularisation.

The classic positron emission tomography (PET) methods of preoperative prediction

\section{KEY MESSAGES}

What is already known about this subject? Perfusion-metabolism mismatch pattern on positron emission tomography (PET) is one of the gold standards for predicting and detecting hibernating myocardium. However, this requires both a perfusion marker and a metabolism marker. The perfusion marker is a short-acting agent necessitating the presence of a cyclotron on site. In addition, there are occasional difficulties in ensuring the superimposition of the perfusion and the metabolism scans. We have already demonstrated the reliability of the ECG-gated metabolic scans in assessing myocardial contraction and thickening in comparison to the cine cardiac MRI scans. Finally, several investigators have confirmed that the presence of metabolic activity in a myocardial segment beyond a threshold of usually $50 \%$ is associated with viability.

What does this study add?

- The possibility of producing two sets of data from using a single marker to define both metabolic and mechanical activity of the myocardium.

- The predictability of the PET gold standard marker of hibernating myocardium using ECGgated ${ }^{18} \mathrm{~F}$-fluorodeoxyglucose-PET.

How might this impact on clinical practice?

- The possibility of performing tests of myocardial hibernation using a long-acting metabolic markers in centres where a cyclotron is not available on site, if the hypothesis is proven prospectively.

- New definition of hibernating myocardium using the mismatch between metabolism and mechanical defects when proven could be more representative of the pathophysiology particularly given the continuing dilemma of whether hibernating myocardium suffers from chronic hypoperfusion or repetitive stunning.

- However, these benefits could not be realised until the hypothesis is proven prospectively.

of viable and hibernating myocardium is the presence of perfusion-metabolism mismatch pattern. ${ }^{4}$ This uses dual radioisotope imaging 
technique. We are reporting the retrospective testing of the predictability of the perfusion-metabolism mismatch pattern, through the use of ECG-gated metabolic myocardial images, thus using a single radio-isotope.$^{56}$ The new method relies on combining information on myocardial metabolism and thickening. We propose that impaired but viable myocardium could be probably predicted by demonstrating continuing metabolic activity in a region with reduced myocardial thickening (metabolic-mechanical mismatch pattern).

\section{METHODS}

\section{Subjects}

The patients were consecutive patients attending the John Mallard Scottish PET Centre for the detection of myocardial viability. The patients underwent the PET studies investigating the presence of hibernating myocardium in more than one study approved by the ethical committee. All the patients contributing to this investigation were in sinus rhythm (for feasibility of ECG gating), and had severe left ventricular systolic impairment, with left ventricular ejection fraction $<35 \%$ as measured by modified Simpson method. All the patients had ischaemic heart disease causing their left ventricular impairment.

\section{Ethical committee approval}

The patients gave informed written consent before undergoing PET. The imaging protocol was approved by the local research ethics committee.

\section{Positron emission tomography}

All the patients had perfusion and metabolic imaging using PET. The perfusion assessment used ${ }^{13} \mathrm{~N}$-ammonia $\left({ }^{13} \mathrm{~N}-\mathrm{NH}_{3}\right) .{ }^{7}$ The metabolism assessment used ${ }^{18} \mathrm{~F}-2$ fluoro-2-deoxyglucose $\left({ }^{18} \mathrm{~F}\right.$-FDG $),{ }^{8}$ which was acquired using an ECG-gated mode. The data were then re-visited and divided into two sets of data for each patient: the first set was combined perfusion-metabolism data and the second set was the combined data of metabolism and wall thickening. The latter was gained by acquiring the ${ }^{18} \mathrm{~F}$-FDG images in an ECG-gated mode.

Tomographic imaging was performed using a Siemens ECAT EXACT 31 PET scanner, which produces 31 slices, the slice separation is $3 \mathrm{~mm}$ and the reconstructed image resolution is $10 \mathrm{~mm}$. Attenuation correction is achieved by a transmission scan using three revolving rod sources prior to the emission scan.

${ }^{13} \mathrm{~N}^{-\mathrm{NH}_{3}}$ was given intravenously at a dose of $370 \mathrm{MBq}$ $(1.5 \mathrm{mSv})$. Volume images of myocardial uptake were then gathered up to 20 min after injection.

${ }^{18}$ F-FDG was injected intravenously at a dose of 185 $\mathrm{MBq}(0.5 \mathrm{mSv}), 1$ hour following an oral glucose load of $50 \mathrm{~g}$ of glucose. ${ }^{9}$ Patients with diabetes received 4-10 units of intravenous soluble insulin to keep their serum glucose level between 4 and $11 \mathrm{mmol} / \mathrm{L}$. The patient was positioned with careful attention to alignment based on markers put on the chest wall and using laser beams created by the scanner at the time of positioning; we were able to ensure that the patient's position was the same during both scans. ${ }^{10}$ The ${ }^{18}$ F-FDG uptake images were acquired $60 \mathrm{~min}$ postinjection.

\section{The ECG-gated acquisition of data}

The ${ }^{18}$ F-FDG uptake images were acquired in an ECG-gated mode, with imaging data gathered at the R wave. ${ }^{56}$ Wall thickening assessment to produce regional wall motion analysis by ECG-gated ${ }^{18} \mathrm{~F}$-FDG was done in the following fashion: myocardial uptake was measured by reforming the ventricle into four short-axis sections covering the base to mid-ventricle. The apex to mid-ventricle was reformed as eight long-axis sections at $22.5^{\circ}$ intervals round the ventricle's long axis. Radial profiles were formed at $22.5^{\circ}$ intervals round each short-axis section producing 16 profiles for each short-axis section. Five radial profiles were formed at $30^{\circ}$ intervals from each of the long-axis sections. This three-dimensional sampling profile set more closely reflects the most likely wall thickening direction than simple short-axis sampling. A complete set of wall profiles was obtained at each of eight phases using a fixed laboratory frame of reference. Each profile was analysed to obtain a hybrid wall thickness parameter given by the product of maximum wall uptake in the profile and the 'thickness' of the wall as given by the second moment of the radial profile. Use of the second moment of radial profile allows for partial volume effect. ${ }^{11}$ The product of uptake and apparent thickness has been shown to be more linearly related to actual wall thickness than the individual parameters. ${ }^{12}$ The time variation in hybrid wall thickness for each profile direction was Fourier analysed to obtain an amplitude and phase value. The amplitude was subsequently converted into a percentage wall thickening value. The variation in wall thickening was converted into a polar map representation.

\section{Production of polar maps}

The reconstructed images were rotated and re-sliced to generate short-axis sections of the myocardium, which were subsequently analysed using circumference profile analysis to produce polar maps diagrammatically showing the myocardial distribution of the tracers (figure 1). ${ }^{11}$ The left ventricle as represented by the polar map was divided into two regions: anterior and inferior.

\section{Determination of the perfusion-metabolism mismatch pattern}

The myocardial region with the highest uptake of ${ }^{13} \mathrm{~N}-$ $\mathrm{NH}_{3}$ was taken as the reference ${ }^{13} \mathrm{~N}-\mathrm{NH}_{3}$ level for that patient. The abnormally perfused areas were semiquantitatively compared with the reference area. The glucose uptake in that reference area was regarded as the reference ${ }^{18} \mathrm{~F}$-FDG level of uptake for that particular patient. The rest of the myocardium was then compared with this reference level. 

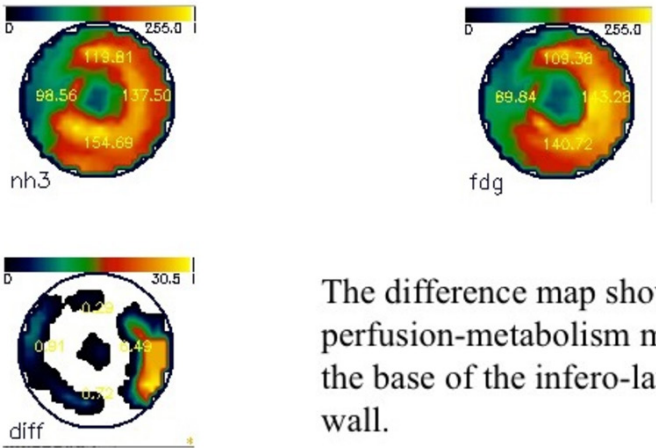

The difference map showing the perfusion-metabolism mismatch in the base of the infero-lateral wall.

Figure 1 The figure shows three polar maps. (1) Perfusion polar map using ${ }^{13} \mathrm{~N}$-ammonia showing severe reduction of perfusion in the septum and the apex. There is a relatively mildly reduced perfusion in the lateral wall. (2) Metabolism polar map using ${ }^{18} \mathrm{~F}$-fluorodeoxyglucose (FDG) showing the glucose uptake is particularly raised in the base of the inferolateral wall, resulting in (3) the difference map showing the perfusion-metabolism mismatch in the base of the inferolateral wall.

Areas of the myocardium with perfusion-metabolism mismatch pattern were thus identified. ${ }^{2}$ The assessment of mismatch pattern was carried out qualitatively by simultaneous inspection of perfusion and metabolism images.

Determination of metabolism-mechanical mismatch pattern The region with the best degree of wall thickening is considered as the reference region for wall thickening, and its ${ }^{18} \mathrm{~F}$-FDG uptake was considered as the reference level of ${ }^{18} \mathrm{~F}$-FDG uptake. It is known that areas with ${ }^{18} \mathrm{~F}$ FDG uptake higher than $50 \%$ of the reference area are viable. ${ }^{13}$ Subsequently, the wall thickening of these regions was then determined. Metabolic-mechanical mismatch pattern was considered to be present if the ${ }^{18}$ F-FDG uptake was higher than $50 \%$ of the reference area with severely reduced $(<50 \%$ of the normal wall thickening) or absent wall thickening. The latter could be semiquantitatively assessed by taking a wall thickening threshold of $50 \%$ of the thickening in the reference area, as the level below which the contraction is taken to be significantly impaired (figure 2). The reliability of gated ${ }^{18}$ F-FDG imaging as a method of assessing myocardial thickening and therefore reflecting the mechanical aspect of myocardial function has been confirmed by our group. ${ }^{6}$

\section{Comparison between the two data sets}

The two data sets were then compared with look at the concordance of the results, and the predictability of the perfusion-metabolism mismatch pattern, by using the metabolism-mechanical mismatch pattern as surrogates.

\section{Statistics}

Continuous variables are presented as mean $\pm \mathrm{SD}$. Categorical data are presented as frequencies. Data were analysed using SPSS for Microsoft Windows Release V. 6.1 software package.
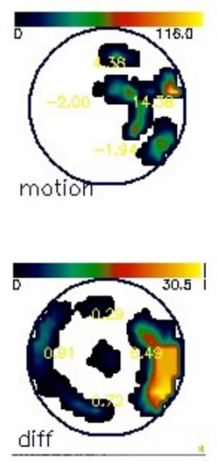

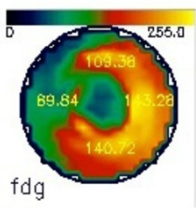

The difference map showing the perfusion-metabolism mismatch in the base of the infero-lateral wall, is identical to the difference between the metabolic and motion maps.

Figure 2 The motion map (1) shows that there is profound reduction of motion in most of the left ventricle, in keeping with severe left ventricular impairment particularly in the base of the infero-lateral wall. The metabolic map (2) shows that glucose uptake is particularly raised in the base of the infero-lateral wall. The difference map (3) shows the mechanical metabolic mismatch pattern very similar to that of the perfusion-metabolism mismatch in the base of the infero-lateral wall.

\section{RESULTS}

There were 55 patients (48 males) in sinus rhythm, with a mean \pm SD age of $62.4 \pm 8.6$ years, with confirmed ischaemic heart disease as the aetiology for the left ventricular systolic impairment. The latter was based on the measurement of left ventricular ejection fraction using the modified Simpson's method at $<35 \%$. The risk factors' profile of the patients is shown in table 1. Of the 55 patients included in the study, data on their coronary angiograms revealed occlusion of one or more coronary artery in 40 patients. Not all of these occlusions were associated with a clear history of acute coronary syndrome that the patient had recalled. Of the remaining patients, four patients had a definite ST elevation myocardial infarction (STEMI) in the past treated at the time with thrombolysis. There was no definite history of STEMI in the remaining 11 patients.

The intra-observer variability was $94 \%$. The two observers who determined the defects, resolved their differences by consensus.

Perfusion-metabolism mismatch pattern was present in 26 patients. The ECG-gated ${ }^{18}$ F-FDG images revealed that 25 patients had metabolism-mechanical mismatch pattern. Two of the patients with perfusion-metabolism mismatch pattern did not have concordant metabolism-mechanical mismatch pattern. On the other hand, one patient with metabolism-mechanical mismatch pattern did not have a concordant perfusion-metabolism mismatch pattern.

At regional level, there were 26 patients with perfusion-metabolism mismatch pattern. Of those, two patients did not have concordant metabolism-mechanical mismatch pattern. A 27th patient had metabolism-mechanical mismatch pattern with no perfusion-metabolism mismatch pattern. Of the 24 remaining patients with 


\begin{tabular}{lc}
\hline Table 1 & Patients' characteristics and risk factors \\
\hline Characteristic & Incidence \\
\hline Age (mean \pm SD) & $62.4 \pm 8.6$ years \\
Male gender & $87 \%$ \\
Diabetes mellitus & $16 \%$ \\
Hypertension & $27 \%$ \\
Hypercholesterolaemia & $89 \%$ \\
Positive family history & $55 \%$ \\
\hline Positive smoking history & $76 \%$ \\
\hline
\end{tabular}

concordant perfusion-metabolism mismatch pattern and metabolic-mechanical mismatch pattern scoring, the mismatch area was identical in size in 18 patients. The estimate of the size of the mismatch region was different in six patients. The accuracy of the size of the mismatch pattern may vary between the two methods in up to 6 of the 24 patients with concordant perfusion-metabolism and metabolism-mechanical mismatch patterns $(25 \%)$. The results taking into consideration the total cohort (those with and without mismatch pattern on perfusion-metabolism assessment by PET) were concordant in 52 of the 55 patients $(95 \%)$.

The metabolism-mechanical mismatch pattern as a surrogate marker for the classical perfusion-metabolism mismatch pattern is sensitive $(92 \%)$ and specific $(97 \%)$. In this cohort, it has excellent positive and negative predictive accuracies: $96 \%$ and $93 \%$, respectively. (figure 3).

\section{DISCUSSION}

Hibernating myocardium is defined by its recovery following revascularisation. ${ }^{14}$ It is advisable to preoperatively detect the presence of hibernating myocardium by an imaging technique in the patients with severe left ventricular impairment, using one of the imaging

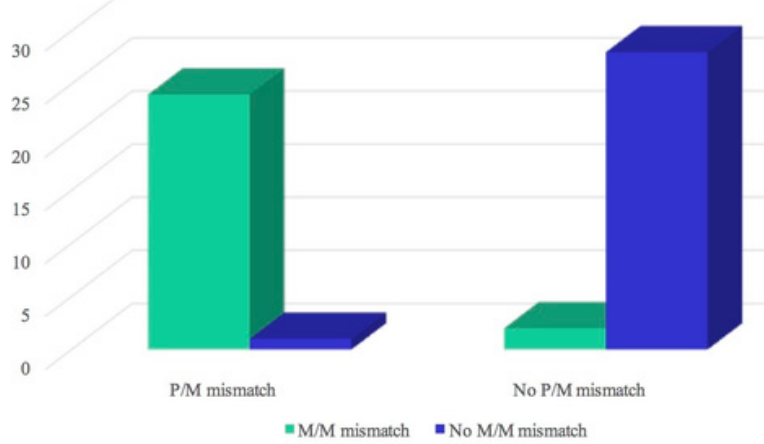

Figure 3 Comparison between the metabolic mechanical imaging and perfusion metabolic imaging in the same patients. The figure demonstrates the high positive and negative predictive values of gated metabolic imaging, in relation to the dual perfusion and metabolic imaging by positron emission tomography. MM, metabolicmechanical mismatch PM, perfusion-metabolic mismatch. techniques. ${ }^{15}$ The PET preoperative detection of hibernating myocardium is one of these techniques. It relies on demonstrating continuing metabolism of glucose in areas of underperfusion, the perfusion-metabolism mismatch pattern. ${ }^{81617}$ Pathophysiologically, this is based on the fact the ischaemic myocardium switches to mainly anaerobic metabolism of glucose in lieu of free fatty acids. ${ }^{18}$

The metabolic PET marker of glucose metabolism is the extrinsic glucose analogue ${ }^{18}$ F-FDG. The use of the combination of perfusion and metabolic imaging, with ${ }^{13} \mathrm{~N}_{-\mathrm{NH}_{3}}$ and ${ }^{18} \mathrm{~F}-\mathrm{FDG}$, respectively, is the most commonly used PET combination to detect hibernating myocardium, ${ }^{19}$ and has high positive and negative predictive accuracies of functional improvement with myocardial revascularisation. ${ }^{81620}$

Allman et al. carried out a meta-analysis of 24 observational studies of 3088 patients and concluded that single-photon emission computed tomography (SPECT) and PET-based imaging have similar abilities in the prediction of survival benefit after revascularisation..$^{21}$ In contrast, Rohatgi et al. showed that myocardial viability was present by PET in $60 \%$ of the myocardial regions classified as scarred by thallium SPECT in patients with ischaemic left ventricular systolic impairment, ${ }^{22}$ and detected a significant improvement in survival after revascularisation in these patients compared with medical treatment alone. ${ }^{22}$

Gated ${ }^{18}$ F-FDG PET is known to be useful in assessing myocardial contraction and left ventricular ejection fraction. ${ }^{23}$ We have previously confirmed its usefulness in this regard when compared with cardiac MRI. ${ }^{6}$

In this study, we propose a new PET-based method for the prediction of hibernating myocardium, which may provide a new definition of the phenomenon. We found that metabolism-mechanical mismatch pattern by gated ${ }^{18}$ F-FDG PET could predict the presence of perfusion-metabolism mismatch pattern with high sensitivity, specificity, positive and negative predictive accuracy. Our study lacked the confirmation of the predictability of hibernation through the demonstration of improved contraction after revascularisation. This study is a retrospective examination of already acquired two sets of data. The latter two are limitations to our study.

The new aspect of our study is the combination of metabolism and mechanical (wall motion and thickness) assessments using a single radiopharmaceutical agent. The proposition is that the metabolism-mechanical mismatch pattern will detect and potentially offer a new definition of hibernating myocardium. The latter would, we recognise, require prospective assessment of the proposed definition using the gold standard for identifying hibernating myocardium which is improved myocardial contraction following revascularisation. If proven, the new definition would also allow the use of ${ }^{18} \mathrm{~F}$ FDG PET in centres where there is no on-site cyclotron, and thus there is no access to the perfusion short-acting PET markers. 
We do recognise, however, that reliance on metabolic imaging by ${ }^{18}$ F-FDG PET is not free from problems related to the difficulties in achieving optimal uptake of the tracer in patients with diabetes. Some argue that in the absence of perfusion data one may have difficulty ascertaining and interpreting the metabolism data. One, however, could argue that this may be related to our collective habit of assessing metabolism in combination with perfusion and it may be that once the new concept had been verified prospectively using the gold standard for the definition of hibernating myocardium, the cohort of those performing this type of cardiac imaging may begin to use the metabolism and the mechanical data in combination in a way similar to our current reliance on perfusion when we assess metabolism data.

In the past, Gerber et al., from the European Community Concerted Action Multicenter study on use of ${ }^{18}$ F-FDG PET for the Detection of Myocardial Viability, confirmed that it is possible to predict myocardial viability by using ${ }^{18}$ F-FDG PET. They used hyperinsulinemic euglycemic clamp and ${ }^{18}$ F-FDG PET prerevascularisation and postrevascularisation (coronary artery bypass grafting (CABG) or angioplasty). The accuracy was $67 \%$, their technique had a high sensitivity of $79 \%$ and a specificity of $55 \%$ when there were at least three dysfunctional myocardial segments with FDG uptake $>45 \%$ of that in the remote normal segments. ${ }^{24}$

Fath-Ordoubadi et al. proposed as a result of their study of hibernating myocardium using quantitative technique of perfusion reserve and metabolic rate of glucose after using the glucose clamp a threshold value for the metabolic rate of glucose of $\geq 0.25 \mu \mathrm{mol} / \mathrm{g} / \mathrm{min}$ that would allow the best prediction of improvement in functional class of at least one grade after revascularisation. ${ }^{25}$

The findings of both Gerber et al. and Fath-Ordoubadi et al. are superior to ours on the basis that they included the ultimate confirmation of the presence of hibernating myocardium by assessing the outcome following revascularisation. Both studies used euglycaemic glucose clamp which improves the quality of images in patients with diabetes. ${ }^{19}$

We do recognise that the interpretation of the metabolic images may be problematic if taken alone with no perfusion imaging, as the behaviour of the myocardial metabolism varies between those with normal myocardial flow and those with ischaemia. When we analysed the metabolism-mechanical data, the analysing investigator was blinded to the perfusion data already collected on the patients.

We also note the entity of reverse perfusion-metabolism mismatch that is rarely seen where the perfusion is not decreased but the metabolism is decreased. The interpretation of the latter is difficult in any case. Such rare cases could be missed if one was using a single metabolic agent (as we proposed), with no perfusion data. This is a potential pitfall.

It is important if this new concept of metabolism-mechanical mismatch pattern was tested prospectively, that an assessment is made of the critical mass of myocardium thus predicted as potentially hibernating necessary to make a clinical difference if the patient was to be revascularised. We do know from other techniques that 5 segments of the 17-segment model of the left ventricular myocardium is regarded as the threshold for revascularisation in these patients to result in an improved clinical outcome. Similar tests need to be carried out if we were testing this new concept.

Our study is simply a pilot study that showed the potential feasibility of an alternative method for the prediction of hibernating myocardium using a single radiopharmaceutical, and an alternative PET definition of hibernating myocardium. If prospectively confirmed by improved contraction after revascularisation, metabolism-mechanical mismatch pattern by ECG-gated ${ }^{18}$ F-FDG PET could provide an easier way to perform PET detection of hibernating myocardium (through access to the technique to centres that do not have a cyclotron on site), and a new PET definition of hibernation linking the absence or significant reduction of mechanical activity to the continuing metabolic activity.

\section{Other potential limitations to the proposed concept}

First, its utility is limited to the patients who are in normal sinus rhythm. Second, the poor myocardial uptake of glucose in patients with diabetes results in much reduced counts when the glucose uptake data are acquired in an ECG-gated mode. This could limit the use of this new diagnostic method in patients with diabetes who constitute around $24 \%$ of the patients with ischaemic heart disease and significant left ventricular systolic impairment, although in this study cohort diabetes mellitus affected only $16 \%$ of the patients.

\section{CONCLUSION}

Metabolism-mechanical mismatch pattern could predict perfusion-metabolism mismatch pattern on PET in patients with myocardial viability. Prospective validation against the gold standard of improved myocardial contraction after revascularisation is needed.

Acknowledgements The authors are grateful for the help from Dr H Ali and Dr A Dawson.

Contributors All the authors have contributed to the research reported and to the production of the manuscript. No one else had materially contributed to this work.

Funding This study was performed using a research grant from the Aberdeen Royal Hospitals Trust's Endowment Fund, with further support from the Department of Medical Physics at the University of Aberdeen, for which the authors express their gratitude.

Competing interests None declared.

Patient consent Obtained.

Ethics approval Grampian Clinical Ethics Committee.

Provenance and peer review Not commissioned; externally peer reviewed.

Open Access This is an Open Access article distributed in accordance with the Creative Commons Attribution Non Commercial (CC BY-NC 4.0) license, which permits others to distribute, remix, adapt, build upon this work non-commercially, and license their derivative works on different terms, provided the original work 
is properly cited and the use is non-commercial. See: http://creativecommons.org/ licenses/by-nc/4.0/

(c) Article author(s) (or their employer(s) unless otherwise stated in the text of the article) 2017. All rights reserved. No commercial use is permitted unless otherwise expressly granted.

\section{REFERENCES}

1. Cowie MR, Wood DA, Coats AJ, et al. Survival of patients with a new diagnosis of heart failure: a population based study. Heart 2000;83:505-10.

2. al-Mohammad A, Mahy IR, Norton MY, et al. Prevalence of hibernating myocardium in patients with severely impaired ischaemic left ventricles. Heart 1998;80:559-64.

3. Petrie MC, Jhund PS, She L, et al. Ten-Year Outcomes after coronary artery bypass grafting according to Age in Patients with Heart failure and left ventricular systolic dysfunction: an analysis of the extended Follow-Up of the STICH trial (Surgical treatment for ischemic Heart failure). Circulation 2016;134:1314-24.

4. Pagano D, Bonser RS, Townend JN, et al. Predictive value of dobutamine echocardiography and positron emission tomography in identifying hibernating myocardium in patients with postischaemic heart failure. Heart 1998;79:281-8.

5. Yamashita K, Tamaki N, Yonekura Y, et al. Regional wall thickening of left ventricle evaluated by gated positron emission tomography in relation to myocardial perfusion and glucose metabolism. $J ~ N u c l$ Med 1991;32:679-85.

6. Waiter GD, Al-Mohammad A, Norton MY, et al. Regional myocardial wall thickening assessed at rest by ECG gated ${ }^{(18)}$ F-FDG positron emission tomography and by magnetic resonance imaging. Heart 2000;84:332-3.

7. Schelbert HR, Phelps ME, Hoffman EJ, et al. Regional myocardial perfusion assessed with $\mathrm{N}-13$ labeled Ammonia and positron emission computerized axial tomography. Am J Cardiol 1979;43:209-18.

8. vom Dahl J, Eitzman DT, al-Aouar ZR, et al. Relation of regional function, perfusion, and metabolism in patients with advanced coronary artery disease undergoing surgical revascularization. Circulation 1994:90:2356-66.

9. Al-Mohammad A, Norton MY, Mahy IR, et al. Can the surface electrocardiogram be used to predict myocardial viability? Heart 1999;82:663-7.

10. Egred M, Al-Mohammad A, Waiter GD, et al. Detection of scarred and viable myocardium using a new magnetic resonance imaging technique: blood oxygen level dependent (BOLD) MRI. Heart 2003;89:738-44.

11. Goris ML, Thompson C, Malone LJ, et al. Modelling the integration of myocardial regional perfusion and function. Nucl Med Commun 1994;15:9-20.
12. Buvat I, Bartlett ML, Kitsiou AN, et al. A "hybrid" method for measuring myocardial wall thickening from gated PET/SPECT images. J Nucl Med 1997;38:324-9.

13. Chan RK, Lee KJ, Calafiore $P$, et al. Comparison of dobutamine echocardiography and positron emission tomography in patients with chronic ischemic left ventricular dysfunction. J Am Coll Cardiol 1996;27:1601-7.

14. Rahimtoola SH. The hibernating myocardium. Am Heart $J$ 1989:117:211-21.

15. Campbell F, Thokala P, Uttley LC, et al. Systematic review and modelling of the cost-effectiveness of cardiac magnetic resonance imaging compared with current existing testing pathways in ischaemic cardiomyopathy. Health Technol Assess 2014;18:1-120.

16. Marwick TH, Maclntyre WJ, Lafont A et al. Metabolic responses of hibernating and infarcted myocardium to revascularization. A followup study of regional perfusion, function, and metabolism. Circulation 1992;85:1347-53.

17. Schelbert HR. Positron emission tomography for the assessment of myocardial viability. Circulation 1991;84:I122-31.

18. Gropler RJ, Geltman EM, Sampathkumaran K, et al. Functional recovery after coronary revascularization for chronic coronary artery disease is dependent on maintenance of oxidative metabolism. J Am Coll Cardiol 1992;20:569-77.

19. Camici PG, Prasad SK, Rimoldi OE. Stunning, hibernation, and assessment of myocardial viability. Circulation 2008;117:103-14.

20. Tamaki N, Yonekura Y, Yamashita K, et al. Prediction of reversible ischemia after coronary artery bypass grafting by positron emission tomography. J Cardiol 1991;21:193-201.

21. Allman KC, Shaw LJ, Hachamovitch R, et al. Myocardial viability testing and impact of revascularization on prognosis in patients with coronary artery disease and left ventricular dysfunction: a metaanalysis. J Am Coll Cardiol 2002;39:1151-8.

22. Rohatgi R, Epstein S, Henriquez J, et al. Utility of positron emission tomography in predicting cardiac events and survival in patients with coronary artery disease and severe left ventricular dysfunction. Am J Cardiol 2001;87:1096-9.

23. Willemsen AT, Siebelink HJ, Blanksma PK, et al. Automated ejection fraction determination from gated myocardial FDG-PET data. $\mathrm{J} \mathrm{Nucl}$ Cardiol 1999;6:577-82.

24. Gerber BL, Ordoubadi FF, Wijns W, et al. Positron emission tomography using(18)F-fluoro-deoxyglucose and euglycaemic hyperinsulinaemic glucose clamp: optimal criteria for the prediction of recovery of post-ischaemic left ventricular dysfunction. results from the European Community Concerted Action Multicenter study on use of(18)F-fluoro-deoxyglucose Positron Emission Tomography for the detection of myocardial viability. Eur Heart $J$ 2001;22:1691-701.

25. Fath-Ordoubadi F, Beatt KJ, Spyrou N, et al. Efficacy of coronary angioplasty for the treatment of hibernating myocardium. Heart 1999;82:210-6. 